Canadian

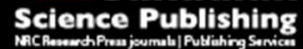

Canadian Journal of Microbiology Revue canadienne de de microbiologie

\title{
Microbiome Profiling of Drinking Water in Relation to Incidence of Inflammatory Bowel Disease
}

\begin{tabular}{|r|l|}
\hline Journal: & Canadian Journal of Microbiology \\
\hline Manuscript ID & cjm-2016-0219.R1 \\
\hline Danuscript Type: & Article \\
\hline Complete List of Authors: & $\begin{array}{r}\text { Forbes, Jessica; University of Manitoba, Medical Microbiology and Infectious } \\
\text { Diseases; National Microbiology Laboratory, Public Health Agency of } \\
\text { Canada } \\
\text { Van Domselaar, Gary; University of Manitoba, Medical Microbiology and } \\
\text { Infectious Diseases; National Microbiology Laboratory, Public Health } \\
\text { Agency of Canada } \\
\text { Sargent, Michael; Department of Internal Medicine and the University of } \\
\text { Manitoba IBD Clinical and Research Centre } \\
\text { Green, Chris; University of Manitoba, Department of Community Health } \\
\text { Sciences } \\
\text { Springthorpe, Susan; University of Ottawa, Centre for Research on } \\
\text { Environmental Microbiology } \\
\text { Krause, Denis; University of Manitoba, Medical Microbiology and Infectious } \\
\text { Diseases } \\
\text { Bernstein, Charles; Department of Internal Medicine and the University of } \\
\text { Manitoba IBD Clinical and Research Centre }\end{array}$ \\
\hline Keyword: & $\begin{array}{l}\text { water microbiome, inflammatory bowel disease, epidemiology, 16s rDNA, } \\
\text { etiology }\end{array}$ \\
\hline
\end{tabular}


1 Microbiome Profiling of Drinking Water in Relation to Incidence of Inflammatory Bowel Disease

2

3 Jessica D. Forbes ${ }^{1,2}$, Gary Van Domselaar ${ }^{1,2}$, Michael Sargent $^{3}$, Chris Green ${ }^{4}$, Susan Springthorpe ${ }^{5}$,

4 Denis O. Krause ${ }^{1,+}$, Charles N. Bernstein ${ }^{3, *}$

\section{Author Affiliations}

$7 \quad{ }^{1}$ Department of Medical Microbiology and Infectious Diseases, University of Manitoba, Canada

$8{ }^{2}$ National Microbiology Laboratory, Public Health Agency of Canada, Winnipeg, Manitoba, Canada

$9{ }^{3}$ Department of Internal Medicine and the University of Manitoba IBD Clinical and Research Centre,

10 Winnipeg, Manitoba, Canada

$11{ }^{4}$ Department of Community Health Sciences, Faculty of Medicine, University of Manitoba, Canada

$12{ }^{5}$ Centre for Research on Environmental Microbiology, University of Ottawa, Ottawa, ON

$13{ }^{\dagger}$ Deceased 16 October 2011.

$14{ }^{*}$ Corresponding author: Charles N. Bernstein Charles.Bernstein@umanitoba.ca,804F-715 McDermot

15 Avenue, Winnipeg, Manitoba, Canada R3E3P4, Phone: (204) 789-3369, Fax: (204) 789-3972

16 Jessica D. Forbes umforb25@myumanitoba.ca;

17 Michael Sargent Michael.Sargent@umanitoba.ca;

18 Gary Van Domselaar Gary.VanDomselaar@phac-aspc.gc.ca;

19 Chris Green chris.green.wpg@gmail.com;

20 Susan Springthorpe spring@uottawa.ca 


\section{$21 \quad$ ABSTRACT}

22 The etiology of IBD is unknown; current research is focused on determining environmental factors.

23 One consideration is drinking water: water systems harbour a considerable diversity of microbes with

24 bacterial concentrations estimated at $10^{6}-10^{8}$ cells per liter. Perhaps differences in microbial ecology

25 of water sources may impact on differential incidence rates of IBD. Regions of Manitoba were

26 geographically mapped according to incidence rates of IBD and identified as high (HIA) or low (LIA)

27 incidence areas. Bulk water, filter material and pipe wall samples were collected from public buildings

28 in different jurisdictions and their population structure analyzed using $16 \mathrm{~S}$ rDNA sequencing. At the

29 phylum level Proteobacteria were observed significantly less frequently $(P=0.02)$ in HIA versus LIA.

30 The abundance of Proteobacteria was also found to vary according to water treatment distribution

31 networks. Gammaproteobacteria was the most abundant class of bacteria and was observed more

32 frequently $(P=0.006)$ in LIA. At the genus level, microbes found to associate with HIA include

33 Bradyrhizobium $(P=0.02)$ and Pseudomonas $(P=0.02)$. Particular microbes were found to associate

34 with LIA or HIA, based on sample location and/or type. This work lays out a basis for further studies

35 exploring water as a potential environmental source for IBD triggers.

\section{Keywords}

38 Inflammatory bowel disease, 16S rDNA, water microbiome, etiology, epidemiology

List of Abbreviations

41 IBD - inflammatory bowel disease; HIA - high incidence area; LIA - low incidence area; UMIBDED -

42 University of Manitoba IBD Epidemiology Database; CD - Crohn's disease; UC - ulcerative colitis; OTU 43 - operational taxonomic unit 


\section{4}

45 Competing Interests

46 Dr Bernstein is supported in part by the Bingham Chair in Gastroenterology. In the past year he has

47 consulted to Abbvie Canada, Shire Canada, Takeda Canada, Theradiag, Cubist Pharmaceutical and

48 Mylan Pharmaceutical. He has received educational grants from Abbvie Canada, Janssen Canada,

49 Shire Canada and Takeda Canada. The other authors have no competing interests to declare. 
INTRODUCTION

51 Canada has among the highest reported incidence rates of inflammatory bowel disease (IBD) worldwide (Molodecky et al. 2012). There are an estimated 250,000 Canadians suffering from IBD (http://cdhf.ca). The first population based incidence rates from Canada were reported from the province of Manitoba, a central province with a population of 1.27 million in 2013 (Bernstein et al. 1999). Capitalizing on universal health care provided within each province and the administrative health databases established within each province, investigators have shown that these high rates in Manitoba are comparably elevated in at least 5 of the other 9 provinces (Bitton et al. 2014; Bernstein et al. 2006).

IBD is an idiopathic, multifaceted disease linked to genetic, immunological and notably, environmental elements. Possible environmental contributions include diet (Vagianos et al. 2016), stress (Iglesias-Rey et al. 2014), medications (Osterman et al. 2014) and smoking (Parkes et al. 2014). A number of factors appear to play a role in influencing the clinical course of disease, however, there is a paucity of data establishing a clear cause-and-effect relationship with IBD.

Many acute illnesses may be secondary to the consumption of contaminated drinking water including legionellosis (Cohn et al. 2014), cholera (Alam et al. 2014) and gastroenteritis (Beaudeau et al. 2014). It is unknown if immunocompetent hosts can acquire chronic diseases like IBD through microbes present in drinking water. Linkages between the development of IBD and specific microorganisms have been investigated such as Mycobacterium paratuberculosis (Rhodes et al. 2013; Suwandi et al.

71 2014) or adherent invasive Escherichia coli (Kotlowski et al. 2007; Agus et al. 2014). In the past decade studies have focused on exploring the gut microbiome using molecular techniques for microorganisms 
73 that may either be protective or injurious to the healthy gut and associated with one form of IBD or

74 another (Balzola et al. 2011; Frank et al. 2007).

While it is theoretically compelling that any or all components of the environment could be involved

77

in pathogen exposure, one realistic and universal geographically-based component is drinking water. The average Canadian adult consumes 1.5 litres of water daily (http://www.hc-sc.gc.ca). Studies have suggested a risk of developing IBD to be associated with iron content of drinking water (Aamodt et al. 2008) as iron may impact the microbial ecology of the human gut (Dostal et al. 2012). However, the concept of the drinking water microbiome as an etiological source of IBD is novel and may provide rationale to the varied geographical distribution of incidence rates observed globally. Drinking water systems harbor a vast diversity of microbes (Hammes et al. 2008; Navarro-Noya et al. 2013); bacterial concentrations of drinking water are estimated at $10^{6}-10^{8}$ cells per liter (Hammes et al. 2008; Navarro-Noya et al. 2013; Lautenschlager et al. 2010). Modern water treatment systems apply filtration and disinfection methods to purify drinking water and inactivate bacteria though it is not possible to extricate all microbes from drinking water distribution systems due to 1) microbes' survival and persistence including biofilm formation and nitrification and 2) inadequate disinfection strategies and a lack of understanding of microbial ecology (Berry et al. 2006). Bacterial communities of drinking water systems may be innocuous, may benefit human health or may be detrimental if harbouring potential pathogen (Berry et al. 2006; Thomas and Ashbolt 2011), therefore it is critical to determine the identity of bacteria present in water distribution systems. Further, microorganisms that are not considered pathogenic may be injurious in specific hosts whose intestinal immune system may be genetically programmed to react aberrantly to them. 
96 The primary goal of this study was to investigate whether the microbial communities of bulk drinking

97 water differs between high (HIA) and low incidence areas (LIA) of IBD and to what extent. The

98 identification of novel microbes widely present in HIA while absent or less frequent in LIA, or

99 alternatively, microbes present in LIA that are absent in HIA may aid in understanding of the etiology

100 of IBD. Secondly, we explored the microbial populations of different sample material including bulk

101 drinking water, filters, and pipe wall (biofilm). Lastly, we assessed microbial communities among a

102 number of sample locations and their associated water distribution system. Herein, we perform a

103 community structure analysis of a number of aspects of the water microbiota to provide comparative

104 associations between microbial community composition and abundance. To our knowledge this work

105 represents the first study investigating the link between the microbiota of drinking water and its

106 association with IBD. 


\section{MATERIALS AND METHODS}

\section{Water supply}

109 The city of Winnipeg receives its water from Shoal Lake, a large isolated lake in southeastern

110 Manitoba (http://winnipeg.ca/waterandwaste/). It is 137 kilometers from Winnipeg and

111 approximately 92 metres higher. Water flows via gravity through the aqueduct to Deacon Reservoir, a

112 four-cell open reservoir on the eastern side of Winnipeg. The City of Winnipeg's water treatment

113 process includes dissolved air flotation, ozonation, filtration and ultraviolet light disinfection. Fluoride

114 and orthophosphate are added then water flows from the water treatment plant to one of three

115 regional reservoirs and pumping stations. Chlorine is added and water flows through the distribution

116 system. Brandon, Manitoba a city of approximately 50,000 is 199 kilometers west of Winnipeg, uses

117 water from the Assiniboine River as a source for its treatment plant; Steinbach, Manitoba, a city of

118 approximately 14,000 is 58 kilometers southeast of Winnipeg uses groundwater as its drinking source.

119 Both rural communities employ water disinfection strategies similar to those just described; however,

120 the key difference is the source of the drinking water.

122 Public buildings were used as sample locations as they were a source of high consumption of water.

123 Verbal permission was obtained from personnel at each location. Each location was equipped with a

124 water filtration system and three pieces of PVC piping. The PVC piping was used to collect biofilm that 125 naturally occurs in drinking water pipes. The water filters were changed every 3 months and the PVC 126 replaced every 4 months over the course of 1 year, providing replicates for each sampling location.

127 Samples were collected in 2005 from HIA $(n=20)$ and LIA $(n=20)$ across Winnipeg, Brandon and 128 Steinbach. We collected samples from 3 water distribution networks (Shoal Lake ( $\mathrm{n}=12$ ), Brandon $129(n=4)$ and Steinbach $(n=4))$. Of the water distributed to Winnipeg areas, samples were obtained from 
1305 reservoirs - Shoal Lake Intake $(n=3)$, Deacon $(n=3)$, McLean $(n=5)$, McPhillips $(n=14)$ and Wilkes

$131(n=7)$. Sample types used in this analysis include, bulk drinking water $(n=8)$, filter $(n=15)$ and pipe wall

132 material $(n=17)$. Sample meta-data can be found in Supplementary Table 1. Samples were removed

133 from the installed PVC system one section at a time. They were placed in zip locked sealed bags,

134 shipped to the laboratory and stored at $4^{\circ} \mathrm{C}$ until processing.

135

136

Data sources and sample collection

137 Our group previously identified HIA and LIA using the University of Manitoba IBD Epidemiology

138 Database (UMIBDED; Figure 1) (Green et al. 2006); the incidence of HIA was $\geq 2$-fold when compared

139 to LIA. The UMIBDED is a population-based archive dating back to 1984 as described in detail

140 elsewhere (Bernstein et al. 2006); it was created from the Manitoba Health databases and includes all

141 Manitobans who meet a validated administrative case definition for IBD.

142

143 Incidence rates were calculated for 2001 for the purpose of this study. We have reassessed small area

144 incidence rates for 2009-2010 and they are minimally changed from 2001 (data not shown). Hence,

145 we anticipate HIA and LIA remained as such in 2005 when water samples were collected. It is worth

146 noting that based on epidemiological surveillance, LIA of Crohn's disease (CD) correlate highly with LIA

147 of ulcerative colitis (UC), with a similar trend observed in HIA.

148

DNA extraction

150 The following nucleic acid extraction protocol was implemented for optimal DNA recovery as the

151 biomass of water was expected to be low: $1 \mathrm{ml}$ of each sample was inoculated in $9 \mathrm{ml} 2.5 \%$ LB broth

152 and incubated aerobically at $37^{\circ} \mathrm{C}$ until slightly turbid. The culture suspension was used for genomic 
153 DNA extraction with Zymo Research Fecal DNA Kits. The DNA concentration and purity were assessed 154 by microspectrophotometry (Beckman DU/800; Beckman Coulter, Inc., Fullerton, CA). Genomic DNA 155 samples were diluted to a concentration of $20 \mathrm{ng} / \mu \mathrm{l}$.

156

157 Sequencing

158 The bacterial 16S rRNA genes were amplified with primers 28F (5'-GAGTTTGATCNTGGCTCAG-3') and 159 519R (5'-GTNTTACNGCGGCKGCTG-3') targeting the V1-V3 hypervariable region (approximately 510 bp). Pyrosequencing was conducted at Research and Testing Laboratory (Lubbock, TX; http://Researchandtesting.com) using a 454 GS FLX (454 Life Sciences, a Roche Company, Brandford, CT) and titanium technology. Sequencing generated 110,760 raw reads.

Comparative sequence analysis

165 Quality control and taxonomic profiling was conducted using mothur (v.1.34.0) (Schloss et al. 2009), a 166 microbial community software program. Barcodes and primers were removed and low quality reads were filtered based on an average quality score $<20$, having read lengths $<210 \mathrm{bp}$, containing hompolymers $>8$ nucleotides and containing any ambiguous base calls. Reads were aligned against the 16S rDNA SILVA database (Pruesse et al. 2007). Sequencing noise was reduced by clustering reads that differ by only $1 \mathrm{bp}$. Chimeric sequences were detected using mothur's implementation of UCHIME and removed (Edgar et al. 2011). The remaining reads were binned into species-level ( $\geq 97 \%$ sequence similarity) operational taxonomic units (OTUs) using the average neighbour algorithm, and taxonomic classification was performed using the SILVA database with Ribosomal Database Project 174 taxonomy and a 70\% minimum bootstrap (Wang et al. 2007). 
176 Alpha diversity

177 Species richness estimates and diversity indices were calculated by algorithms implemented in 178 mothur (Schloss et al. 2009).

179

180 Statistical analysis

181 Analyses were executed using the R package (http://www.r-project.org) phyloseq (McMurdie and 182 Holmes 2013). Samples with <500 reads were removed. OTUs were normalized to relative abundance 183 and filtered to include only OTUs with a mean abundance of $>10^{-4}$ across all samples. Statistical 184 significance of community composition differences was tested using customized R-scripts: the 185 Kruskal-Wallis one-way analysis of variance was applied to microbiota data and community estimates 186 to compare similarities. $P$-values were considered significant at $P<0.05$. Visualization of OTU-based 187 community composition differences was employed using the Bray-Curtis dissimilarity index. 


\section{RESULTS AND DISCUSSION}

189

190

191

192

\section{Bacterial Community Characterization}

Filtering out low quality, chimeric and non-bacterial reads generated 83,392 high quality $16 \mathrm{~S}$ rDNA reads with an average amplicon length of $456 \mathrm{bp}$. The sequencing depth was comparable among groups with $2323 \pm 770$ in HIA and $2253 \pm 693$ in LIA. Therefore, with an average of 2287 reads per sample we achieved a fairly deep sequencing depth capturing the majority of bacterial OTUs.

Sequences were clustered into 749 OTUs based on their shared sequence similarity at a $97 \%$ threshold (3\% sequence divergence). Four samples were excluded from statistical analysis due to insufficient read numbers $(<500)$.

The average coverage for OTU characterization based on Good's coverage was 99.1\% (min-90.0\%; max-99.8\%). Richness (Chao1 and ACE) and diversity (Shannon and Simpson) were comparable among incidence areas (Figure 2a) and sample type (Figure 2b). Investigation of the 7 sampling locations identified differences of richness as estimated by ACE ( $P=0.046$; Figure $2 \mathrm{c})$. To further investigate richness estimates and diversity indices, sample locations were divided into three respective water distribution systems: all Winnipeg locations receiving its water from Shoal lake, Brandon (Assiniboine river) and Steinbach (ground-water). Both Chao $(P=0.04)$ and ACE $(P=0.01)$ richness estimators indicate species richness to vary among the different water systems (Figure 2d). For example, species richness was highest in Steinbach followed by Winnipeg (Shoal Lake) and Brandon. Previous studies suggest that the use of chlorination in water treatment processes significantly reduces the number of observed species (Roeselers et al. 2015); chlorination is used in all water treatment distribution systems presented in this study. In terms of IBD incidence, species richness was lowest in Brandon 
210 and highest in Steinbach which were selected communities of HIA and LIA, respectively. It's possible

211 that the use and associated levels of chlorine could have some effect on incidence areas.

\section{Bacterial population comparisons}

214 Bacterial communities of LIA and HIA

215 Sequences were assigned to 10 phyla, of which phylotypes belonging to the Proteobacteria

216 dominated the overall microbial population. Proteobacteria as a principal phylum in drinking water is

217 well documented in the literature (Lautenschlager et al. 2014; Wang et al. 2014; Liu et al. 2014; Lührig

218 et al. 2015). The average relative abundance of Proteobacteria was $92 \%$. This corresponds with

219 related population-based studies whereby the abundance of Proteobacteria can range anywhere from

22044 to 98\% (Wang et al. 2014; Liu et al. 2014; Lührig et al. 2015). The abundance of Proteobacteria was

221 higher in LIA $(94 \% \pm 21)$ in contrast to HIA $(91 \% \pm 22 ; P=0.02$; Figure 3a, Supplementary Table 2).

222 Furthermore, while there was a lack of significant variation of Proteobacteria between sample

223 locations (Supplementary Table 3), there was a significant distinction in abundance between water

224 treatment systems $(P=0.046$; Steinbach $(100 \%)>$ Shoal Lake $(93 \% \pm 20)>$ Brandon $(76 \% \pm 41)$;

225 Supplementary Table 4). Actinobacteria, Firmicutes and Bacteroidetes also represent common phyla.

226 Phyla present at very low amounts included Acidobacteria, Chloroflexi, Fusobacteria, Spirochaetes,

227 TM7 and Verrucomicrobia.

229 In our analysis, Gammaproteobacteria showed an overall dominance of $54 \%$. This is intriguing, as

230 many population-based or environmental studies have reported either Beta- (Pinto et al. 2012; Lin et

231 al. 2014) or Alphaproteobacteria (Gomez-Alvarez et al. 2012; Bai et al. 2013) to outweigh the

232 Gammaproteobacteria population. The abundance of Gammaproteobacteria was found to differ 
233 between IBD incidence areas $(P=0.006)$. Specifically, Gammaproteobacteria was elevated in LIA (77\% $234 \pm 41)$ when compared to HIA $(28 \% \pm 45)$. We observed moderate changes of Alphaproteobacteria $235(P=0.07)$, which were highest in LIA. Investigation of sample type presented an interesting dynamic: 236 the abundance of Alphaproteobacteria $(P=0.055)$ and Betaproteobacteria $(P=0.091)$ seemed to shift 237 based on the sample type (Supplementary Table 5). A noteworthy observation from this study is that 238 these microbial populations are inversely correlated, for example, Alphaproteobacteria (competitive 239 under oligotrophic conditions and degrading complex organic compounds) are more prevalent within 240 pipe walls whereas Betaproteobacteria (fast-grower and nutrient lovers) (Newton et al. 2011) are 241 more prevalent within water and filter material. Rudi et al. (2010) observed a similar phenomenon 242 utilizing a ProteoQuant assay; the authors concluded that the Alpha- and Betaproteobacteria are in 243 competition, independent of the abundance of Gammaproteobacteria. A moderate variation of 244 Betaproteobacteria was observed among sample locations $(P=0.056)$.

246 Few genera were found to correlate with either incidence area; Pseudomonas

247 (Gammaproteobacteria; $P=0.016$ ) was highest in LIA whereas Bradyrhizobium (Alphaproteobacteria; $248 P=0.02$ ) was highest in HIA (Figure 4a). Pseudomonas comprised approximately $48 \%$ of the microbial 249 population. Such a predominance of Pseudomonas is of particular concern as many species belonging 250 to this genus are biofilm formers (Fazli et al. 2014), which therefore lead to an increase in pathogen 251 persistence. Pseudomonas has been implicated in the pathogenesis of IBD; the Pseudomonas 252 fluorescens-associated sequence 12 that encodes for a T cell superantigen is prevalent among CD (38253 60\%) and UC (42\%) (Prideaux et al. 2012; Wei et al. 2002). Huang et al. (2014) reported Pseudomonas 254 aeruginosa to represent $11.92 \%$ of total sequence reads of filtered water and $11.16 \%$ in drinking 255 water; hence, Pseudomonas was found to be resistant to particular disinfection methods due to 
256 unknown mechanisms. Indeed, many studies support these findings in that $P$. aeruginosa is frequently 257 detected in chlorinated drinking water (Lee et al. 2011; Wingender and Flemming 2011). Conversely, 258 Bradyrhizobium has previously been identified to be a dominant member of chlorinated water 259 (Gomez-Alvarez et al. 2012). The opportunistic pathogenic association, if any, of Bradyrhizobium 260 species to human gastrointestinal diseases is not well defined. To our knowledge, Bradyrhizobium has 261 not been implicated in the pathogenesis of IBD; however, Bradyrhizobium enterica is a newly 262 identified bacterium and has been identified among persons with cord colitis following umbilical-cord 263 hematopoietic stem-cell transplantation (Bhatt et al. 2013). It should be mentioned that both 264 Pseudomonas and Bradyrhizobium have been reported to be a common contaminant of next 265 generation sequencing studies, particularly in samples containing low biomass (Salter et al. 2014). To 266 a minor extent, the abundance of Mycobacterium $(P=0.06)$ was higher in HIA. This genus, particularly, 267 M. paratuberculosis is scrutinized in the possible etiology of CD as there are many conflicting reports 268 regarding its relationship to CD (Suwandi et al. 2014, Nazareth et al. 2015). We identified 269 Mycobacterium in only 3 samples, all of which were HIA and recovered from pipe wall material. 270 Though many of the taxa identified in our study do not reach statistical significance with respect to 271 abundance, application of a principal coordinate analysis (Figure 5a) shows that many HIA samples 272 cluster separately from LIA thereby suggesting that the overall microbial population structure varies. 273 The HIA samples form a tighter cluster compared to LIA samples; many HIA samples were comprised 274 of a relatively low number of different OTUs reflecting a more homeogenous microbiota. Conversely, 275 LIA were more heterogeneous typically including several different OTUs.

277 Bacterial communities of bulk water 
278 In bulk water, Proteobacteria represented $81 \%$ of OTUs, Bacteroidetes represented $10 \%$ and

279 Actinobacteria accounted for 9\% ( $P=0.05$; Figure 3b, Supplementary Table 5). Dominant genera

280 (Figure 4b) of bulk water included Pseudomonas (49\%), Janthinobacterium (18\%) and Massilia (13\%).

281 Liu et al. (2014) has previously characterized the bulk water microbiome; they reported Polaromonas 282 (69\%), Sphingomonas (13\%), Acidovorax (5\%) and Janthinobacterium (4\%) to dominate the bulk water 283 microbiota. In our data, Polaromonas, Sphingomonas and Acidovorax were identified, albeit at very 284 low abundances. Navarro-Noya et al. (2013) investigated the bacterial communities of drinking water 285 wells in Mexico and concluded similar findings with respect to the relative observed abundance of 286 Pseudomonas, Janthinobacterium and Massilia. Interestingly, they also reported Perlucidibaca 287 (13.9\%), Rheinheimera (5.9\%), Alkanindiges (2.2\%) and Psychrobacter (2.1\%) to be dominant 288 members of the Gammaproteobacteria. These genera were not observed in our study; we speculate that environmental characteristics such as temperature, in addition to varied water treatment processes explain their absence. Moreover, while Duganella and Flavobacterium were present in our samples, their abundances were less than previously reported (Navarro-Noya et al. 2013).

Bacterial communities of pipe wall (biofilm)

294 In terms of pipe wall (biofilm), Proteobacteria accounted for $90 \%$ of OTUs, and Actinobacteria and 295 Firmicutes represented 5\% of OTUs individually (Supplementary Table 5). Within pipe material, 296 Pseudomonas (31\%), Sphingomonas (17\%) and Methylobacterium (15\%) dominated the microbiota.

297 This supplements previous studies (Liu et al. 2014), which have reported a dominance of 298 Pseudomonas, Sphingomonas and Janthinobacterium. Furthermore, Wang et al. (2014) detected 299 Legionella, Mycobacterium and P. aeruginosa in biofilms of pipe material. While our pipe material 300 samples were devoid of Legionella, the presence (however paltry) of Mycobacterium and 
301 Pseudomonas is promising with respect to their known potential pathogenesis. The identification and 302 dominance of Pseudomonas and Sphingomonas detected in pipe wall biofilms has been previously 303 established (Berry et al. 2006; Simões et al. 2010). Both genera are able to form biofilms allowing 304 persistence in otherwise harsh, oligotrophic (Sphingomonas) conditions. Moreover, Sphingomonas 305 are able to transform between biofilm and planktonic modes that further supports their dominance in 306 bulk water environments (Bereschenko et al. 2010).

\section{Bacterial communities of filter material}

309 Proteobacteria represented $99.7 \%$ of OTUs associated with filter material (Supplementary Table 5). Intriguingly, Actinobacteria accounted for $9 \%$ of OTUs in bulk water yet in filter material their abundance was negligible. A possible explanation for their absence is their planktonic nature and hence do not preferentially attach to surfaces (Newton et al. 2011). Nitrospirae are frequently detected in filters but were absent in our samples (Lautenschlager et al. 2014). The main genera of filter material included Pseudomonas (67\%), Massilia (8\%) and Sphingobium (7\%).

The variation and associated importance of microbial communities between sample types was variability among distinct sample material (Wang et al. 2014; Liu et al. 2014; Lin et al. 2014). In the present study, however, Acidovorax $(P=0.06)$ was present in both water and filter material whereas Deefgea $(P=0.08)$ and Microbacterium $(P=0.08)$ were present in only bulk water samples, thus, no significant microbial population differences were observed between sample types. Conversely, as shown in Figure 5b, principal coordinate analysis implies that the overall microbial populations vary; most of the pipe wall samples clustered together and likewise, a trend can be observed among filter 
324 samples. Pinto et al. (2012) recently reported Acidovorax, Hydrogenophaga and Denitratisoma to be

325 associated with filter effluent and hence less impacted by disinfection processes, though our samples 326 were devoid of the latter two genera.

Bacterial communities of sample locations

329 The microbial community of sample locations indicated minor variability. The abundance of

330 Firmicutes $(P=0.06)$ demonstrated minimal shifts based on location (Figure 3c, Supplementary Table

$3313)$; in the context of genera, the abundance of Exiguobacterium $(P=0.0009)$ and Janthinobacterium

$332(P=0.01)$ were statistically significant among sample locations (Figure 4c). It has been reported that

333 different locations (within the same geographical region and subject to similar environmental

334 exposures) will be comprised of diverse microbial communities (Roeselers et al. 2015). As water flows

335 from one reservoir to another subjected to sequential disinfection methods it would theoretically be 336 expected that bacterial populations would shift in response. However, the shifts observed in our 337 study were not so dramatic as to significantly affect the microbial populations.

\section{Bacterial communities of water treatment distribution networks}

340 As mentioned, in this study there are three water distribution networks. The first receives its water

341 from Shoal Lake intake and includes the additional locations of Deacon, McPhillips, McLean and

342 Wilkes Reservoirs. The second includes the location Brandon, Manitoba and lastly, Steinbach,

343 Manitoba that utilizes water distribution systems previously described. In this regard, the abundance

344 of Proteobacteria ( $P=0.05)$ was highest in LIA Steinbach and lowest in HIA Brandon (Figure 3d,

345 Supplementary Table 4). Brevundimonas $(0.17 \% \pm 0.3 ; P=0.02)$, Stenotrophomonas $(25 \% \pm 47 ; P=0.02)$

346 and Acinetobacter $(0.98 \% \pm 1 ; P=0.03)$ of Proteobacteria were highest in Steinbach (Figure $4 \mathrm{~d})$. The 
347 abundance of Firmicutes $(P=0.05)$ was significantly different among distribution systems, likely due to 348 Staphylococcus, which was highest in Brandon (24\% $\pm 41 ; P=0.01)$. The aforementioned water 349 distribution systems receive water from different sources, consequently it is expected that the 350 microbial populations somewhat differ.

351

352 We acknowledge our study has methodological limitations; we recognize that the absence of water 353 quality characterization including microbe concentration, $\mathrm{pH}$ and levels of chlorine, minerals and trace 354 elements and others is a major shortcoming of this paper. Furthermore, the methods we applied to 355 enrich the samples were selected to help overcome low biomass issues. Culture enriched molecular 356 profiling has to our knowledge not been performed specifically in water but has been explored in 357 other low biomass areas, including the airway of cystic fibrosis (Sibley et al. 2011). This approach may 358 have biased the results to underrepresent some species and may partially account for the high 359 abundance of Proteobacteria. 


\section{CONCLUSION}

361 Of foremost importance, we have demonstrated that microbial communities differ between incidence 362 areas of IBD within Manitoba. This is critical in attempting to elucidate the geographical variability of 363 IBD prevalence and incidence. Furthermore, we demonstrated a difference among collected sample 364 material and sample location.

366 The concept of drinking water potentially influencing the development and/or course of IBD has not 367 previously been recognized. Recently, the water microbiome has been well characterized (Navarro368 Noya et al. 2013; Lautenschlager et al. 2010; Berry et al. 2006; Roeselers et al. 2015; Lautenschlager et 369 al. 2014; Wang et al. 2014; Lührig et al. 2015; Pinto et al. 2012; Lin et al. 2014; Gomez-Alvarez et al. 2012), however this characterization has not extended to plausible etiologies for particular diseases like IBD. Ample literature is available for waterborne acute ailments yet little to no evidence exists for establishing a relationship among drinking water to chronic diseases.

The notion of a dysbiosis in the gastrointestinal tract of IBD patients is well documented and widely accepted among the gastroenterological research community. What remains to be elucidated however is if this dysbiosis is a cause or consequence of disease. In the case of the latter, is the presence of particular microbes within the gastrointestinal tract responsible for instigating a dysbiosis? And how are IBD patients acquiring these microbes? Numerous genera identified in our analysis are potential human pathogens including but not limited to Pseudomonas, Mycobacterium, Stenotrophomonas, Aeromonas, Acinetobacter and Staphylococcus. Therefore, we speculate drinking water may contribute to IBD etiology. 
383 It is important to highlight that the drinking water examined did not harbor potentially unsafe levels 384 of any known pathogenic bacteria and hence even differences in microbial ecology should not be 385 misconstrued as any measure of lack of safety in the drinking water of Manitoba. However, we have 386 uncovered that within the complexity of the microbial ecology of what is considered safe drinking 387 water there are differences in areas supplied by different water sources of varying incidence of IBD. 388 That having been said, we have not proved any causal associations between microbes and IBD, but 389 rather ecological associations. Our intent in this approach was not to be definitive, but instead, to 390 look for trends that could be pursued in the future. This research lays the ground work for further 391 exploration in other HIA and LIA elsewhere in Canada for similar associations as well as determination 392 as to what extent these microorganisms appear in the human gut of affected and unaffected 393 individuals. 


\section{ACKNOWLEDGEMENTS}

395 Dr. Charles Bernstein is supported in part by the Bingham Chair in Gastroenterology. This research

396 was supported in part by operating grants from the Crohn's and Colitis Foundation of Canada and the 397 Broad Foundation for Medical Research.

398

399 Jessica D. Forbes is supported in part by the Mindel and Tom Olenick Research Studentship in 400 Medicine.

401

402 The authors thank Wenhua Tang for performing wet-lab work and Ehsan Khafipour. The authors also 403 thank Kelly Kjartanson from the City of Winnipeg Waterworks Department. 


\section{REFERENCES}

Aamodt, G., Bukholm, G., Jahnsen, J., Moum, B., Vatn, M.H., Lygren, I., Aubert, E., Henriksen, M., Flaaten, B., Schultz, T., Sauar, J., Kjellevold, $\varnothing$., and Stray, N. 2008. The association between water supply and inflammatory bowel disease based on a 1990-1993 cohort study in southeastern Norway. Am. J. Epidemiol. 168(9):1065-1072. doi:10.1093/aje/kwn218. PMID:18801890.

Agus, A., Massier, S., Darfeuille-michaud, A., Billard, E., and Barnich, N. 2014. Understanding host-adherentinvasive Escherichia coli interaction in Crohn's disease: opening up new therapeutic strategies. Biomed. Res. Int. 9:e12714. doi:10.1155/2014/567929. PMID:25580435.

Alam, M.T., Weppelmann, T.A., Weber, C.D., Johnson, J.A., Rashid, M.H., Birch, C.S., Brumback, B.A., Madsen Beau de Rochars, V.E., Glenn, J., and Ali, A. 2014. Monitoring water sources for environmental reservoirs of toxigenic Vibrio cholerae O1, Haiti. Emerg. Infect. Dis. 20(3):356-363. doi:10.3201/eid2003.131293. PMID:24571741.

Bai, Y., Liu, R., Liang, J., and Qu, J. 2013. Integrated metagenomic and physiochemical analyses to evaluate the potential role of microbes in the sand filter of a drinking water treatment system. PLoS One. 8(4):e61011. doi:10.1371/journal.pone.0061011. PMID:23593378.

Balzola, F., Bernstein, C., and Ho, G.T. 2011. A pyrosequencing study in twins shows that gastrointestinal microbial profiles vary with inflammatory bowel disease phenotypes: Commentary. Inflamm. Bowel Dis. Monit. 11:166. 
Beaudeau, P., Schwartz, J., and Levin, R. 2014. Drinking water quality and hospital admissions of elderly people for gastrointestinal illness in Eastern Massachusetts, 1998-2008. Water Res. 52:188-198. doi:10.1016/j.watres.2014.01.005. PMID:24486855.

Bereschenko, L.A., Stams, A.J.M., Euverink, G.J.W., and Van Loosdrecht, M.C.M. 2010. Biofilm formation on reverse osmosis membranes is initiated and dominated by Sphingomonas spp. Appl. Environ. Microbiol. 76(8):2623-2632. doi:10.1128/AEM.01998-09. PMID:20190090.

Bernstein, C.N., Blanchard, J.F., Rawsthorne, P., and Wajda, A. 1999. Epidemiology of Crohn's disease and ulcerative colitis in a central Canadian province: a population-based study. Am. J. Epidemiol. 149(10):916-924. PMID:10342800.

Bernstein, C.N., Wajda, A., Svenson, L.W., MacKenzie, A., Koehoorn, M., Jackson, M., Fedorak, R., Israel, D., and Blanchard, J.F. 2006. The epidemiology of inflammatory bowel disease in Canada: A population-based study. Am. J. Gastroenterol. 101(7):1559-1568. PMID:16863561.

Berry, D., Xi, C., and Raskin, L. 2006. Microbial ecology of drinking water distribution systems. Curr. Opin. Biotechnol. 17(3):297-302. PMID:16701992.

Bhatt, A.S., Freeman, S.S., Herrera, A.F., Pedamallu, C.S., Gevers, D., Duke, F., Jung, J., Michaud, M., Walker, B.J., Young, S., Earl, A.M., Kostic, A.D., Ojesina, A.L, Hasserjian, R., Ballen, K.K., Chen, Y.B., Hobbs, G., Antin, J.H., Soiffer, R.J., Baden, L.R., Garret, W.S., Hornick, J.L., Marty, F.M., and Meyerson, M. 2013. Sequence-based discovery of bradyrhizobium enterica in cord colitis syndrome. N. Engl. J. Med. 369(6):517-528. doi:10.1056/NEJMoa1211115. PMID:23924002. 
Bitton, A., Vutcovici, M., Patenaude, V., Sewitch, M., Suissa, S., and Brassard, P. 2014. Epidemiology of inflammatory bowel disease in Québec: recent trends. Inflamm. Bowel. Dis. 20(10):1770-1776. doi:10.1097/MIB.0000000000000162. PMID:25159452.

Cohn, P.D., Gleason, J.A., Rudowski, E., Tsai, S.M., Genese, C.A., and Fagliano, J.A. 2014. Community outbreak of legionellosis and an environmental investigation into a community water system. Epidemiol. Infect. 143(6):13221331. doi:10.1017/S0950268814001964. PMID:25083716.

Dostal, A., Chassard, C., Hilty, F.M., Zimmermann, M.B., Jaeggi, T., Rossi, S., and Lacroix, C. 2012. Iron depletion and repletion with ferrous sulfate or electrolytic iron modifies the composition and metabolic activity of the gut microbiota in rats. J. Nutr. 142(2):271-277. doi:10.3945/jn.111.148643. PMID:22190022.

Edgar, R.C., Haas, B.J., Clemente, J.C., Quince, C., and Knight, R. 2011. UCHIME improves sensitivity and speed of chimera detection. Bioinformatics. 27(16):2194-2200. doi:10.1093/bioinformatics/btr381. PMID:21700674.

Fazli, M., Almblad, H., Rybtke, M.L, Givskov, M., Eberl, L., and Tolker-Nielsen, T. 2014. Regulation of biofilm formation in Pseudomonas and Burkholderia species. Environ. Microbiol. 16(7):1961-1981. doi:10.1111/14622920.12448. PMID:24592823.

Frank, D.N., St Amand, A.L., Feldman, R.A., Boedeker, E.C., Harpaz, N., and Pace, N.R. 2007. Molecularphylogenetic characterization of microbial community imbalances in human inflammatory bowel diseases. Proc. Natl. Acad. Sci. 104(34):13780-13785. PMID:17699621. 
Gomez-Alvarez, V., Revetta, R.P., and Domingo, J.W.S. 2012. Metagenomic analyses of drinking water receiving different disinfection treatments. Appl. Environ. Microbiol. 78(17):6095-6102. doi:10.1128/AEM.01018-12. PMID:22729545.

Green, C., Elliott, L., Beaudoin, C., and Bernstein, C.N. 2006. A population-based ecologic study of inflammatory bowel disease: Searching for etiologic clues. Am. J. Epidemiol. 164(7):615-623. PMID:16920784.

Hammes, F., Berney, M., Wang, Y., Vital, M., Köster, O., and Egli, T. 2008. Flow-cytometric total bacterial cell counts as a descriptive microbiological parameter for drinking water treatment processes. Water Res. 42(12):269-277. PMID:17659762.

Huang, K., Zhang, X.X, Shi, P., Wu, B., and Ren, H. 2014. A comprehensive insight into bacterial virulence in drinking water using 454 pyrosequencing and Illumina high-throughput sequencing. Ecotoxicol. Environ. Saf. 109:15-21. doi:10.1016/j.ecoenv.2014.07.029. doi:10.1016/j.ecoenv.2014.07.029. PMID:25129220.

Iglesias-Rey, M., Barreiro-de Acosta, M., Caamaño-Isorna, F., Rodríguez. I.V., Ferreiro, R., Lindkvist, B., González, A.L., and Dominguez-Munoz, J.E. 2014. Psychological factors are associated with changes in the health-related quality of life in inflammatory bowel disease. Inflamm. Bowel. Dis. 20(1):92-102. doi:10.1097/01.MIB.0000436955.78220.bc. PMID:24193152.

Kotlowski, R., Bernstein, C.N., Sepehri, S., and Krause, D.O. 2007. High prevalence of Escherichia coli belonging to the B2+D phylogenetic group in inflammatory bowel disease. Gut. 56(5):669-675. PMID:17028128. 
Lautenschlager, K., Boon, N., Wang, Y., Egli, T., and Hammes, F. 2010. Overnight stagnation of drinking water in household taps induces microbial growth and changes in community composition. Water Res. 44(17):4868-4877. doi:10.1016/j.watres.2010.07.032. PMID:20696451.

Lautenschlager, K., Hwang, C., Ling, F., Liu, W.T., Boon, N., Köster, O., Egli, T., and Hammes, F. 2014. Abundance and composition of indigenous bacterial communities in a multi-step biofiltration-based drinking water treatment plant. Water Res. 62:40-52. doi:10.1016/j.watres.2014.05.035. PMID:24937356.

Lee, C.S., Wetzel, K., Buckley, T., Wozniak, D., and Lee, J. 2011. Rapid and sensitive detection of Pseudomonas aeruginosa in chlorinated water and aerosols targeting gyrB gene using real-time PCR. J. Appl. Microbiol. 111(4):893-903. doi:10.1111/j.1365-2672.2011.05107.x. PMID:21794031.

Lin, W., Yu, Z., Zhang, H., and Thompson, I.P. 2014. Diversity and dynamics of microbial communities at each step of treatment plant for potable water generation. Water Res. 52:218-230. doi:10.1016/j.watres.2013.10.071. PMID:24268295.

Liu, G., Bakker, G., Li, S., Vreeburg, J., Verberk, J.Q.J.C., Medema, G., Liu, W.T., and Van Dijk, J.C. 2014. Integral study of microbial ecology of unchlorinated drinking water distribution system: analysis of structure and composition of bacterial communities in bulk water, suspended solids, loose deposits and pipe wall biofilm. Environ. Sci. Technol. 48:5467-5476.

Lührig, K., Canbäck, B., Paul, C.J., Johansson, T., Persson, K.M., and Rådström, P. 2015. Bacterial community analysis of drinking water biofilms in southern Sweden. Microbes Environ. 30(1):99-107. doi:10.1264/jsme2.ME14123. PMID:25739379. 
McMurdie, P.J., and Holmes, S. 2013. Phyloseq: An R package for reproducible interactive analysis and graphics of microbiome census data. PLoS One. 8(4):e61217. doi:10.1371/journal.pone.0061217. PMID:23630581.

Molodecky, N.A., Soon, I.S., Rabi, D.M., Ghali, W.A., Ferris, M., Chernoff, G., Benchimol, E.I., Panaccione, R., Ghosh, S., Barkema, H.W., and Kaplan, G.G. 2012. Increasing incidence and prevalence of the inflammatory bowel diseases with time, based on systematic review. Gastroenterology. 142(1):46-54.e42. doi:10.1053/j.gastro.2011.10.001. PMID:22001864.

Navarro-Noya, Y.E., Suárez-Arriaga, M.C., Rojas-Valdes, A., Montoya-Ciriaco, N.M., Gómez-Acata, S., FernándezLuqueño. F., and Dendooven, L. 2013. Pyrosequencing analysis of the bacterial community in drinking water wells. Microb. Ecol. 66(1):19-29. doi:10.1007/s00248-013-0222-3. PMID:23563631.

Nazareth, N., Magro, F., Appelberg, R., Silva, J., Gracio, D., Coelho, R., Cabral, J.M., Abreu, C., Macedo, G., Bull, T.J., and Sarmento, A. 2015. Increased viability but decreased culturability of Mycobacterium avium subsp. paratuberculosis in macrophages from inflammatory bowel disease patients under Infliximab treatment. Med. Microbiol. Immunol. 204(6):647-656. doi: 10.1007/s00430-015-0393-2. PMID:25702170.

Newton, R.J., Jones, S.E., Eiler, A., McMahon, K.D., and Bertilsson, S. 2011. A Guide to the Natural History of Freshwater Lake Bacteria. Microbiol. Mol. Biol. Rev. 75(1):14-49. doi:10.1128/MMBR.00028-10. PMID:21372319.

Osterman, M.T., Sandborn, W.J., Colombel, J.F., Robinson, A.M., Lau, W., Huang, B., Pollack, P.F., Thakkar, R.B., and Lewis, J.D. 2014. Increased risk of malignancy with adalimumab combination therapy, compared with monotherapy, for Crohn's disease. Gastroenterology. 146(4):941-949.e2. doi:10.1053/j.gastro.2013.12.025.

Parkes, G.C., Whelan, K., and Lindsay, J.O. 2014. Smoking in inflammatory bowel disease: Impact on disease course and insights into the aetiology of its effect. J. Crohn's Colitis. 8:717-725. PMID:24361468. 
Pinto, A.J., Xi, C., and Raskin, L. 2012. Bacterial community structure in the drinking water microbiome is governed by filtration processes. Environ. Sci. Technol. 46(16):8851-8859. doi:10.1021/es302042t.

PMID:22793041.

Prideaux, L., De Cruz P., Ng, S.C., and Kamm, M.A. 2012. Serological antibodies in inflammatory bowel disease. Inflamm. Bowel. Dis. 2012, 18(7):1340-1355. doi:10.1002/ibd.21903. PMID:22069240.

Pruesse, E., Quast, C., Knittel, K., Fuchs, B.M., Ludwig, W., Peplies, J., and Glöckner, F.O. 2007. SILVA: A comprehensive online resource for quality checked and aligned ribosomal RNA sequence data compatible with ARB. Nucleic Acids Res. 35(21):7188-7196. PMID:17947321.

Rhodes, G., Henrys, P., Thomson, B.C., and Pickup, R.W. 2013. Mycobacterium avium subspecies paratuberculosis is widely distributed in British soils and waters: Implications for animal and human health. Environ. Microbiol. 15(10):2761-2774. doi:10.1111/1462-2920.12137. PMID:23648004.

Roeselers, G., Coolen, J., van der Wielen, P.W.J.J., Jaspers, M.C., Atsma, A., de Graaf, B., and Schuren, F. 2015. Microbial biogeography of drinking water: patterns in phylogenetic diversity across space and time. Environ. Microbiol. 17(7):2505-2514. doi:10.1111/1462-2920.12739. PMID:25581482.

Rudi, K., Berg, F., Gaustad, E., Tannes, T., and Vatn, M. 2010. Ratios between Alpha-, Beta- and Gammaproteobacteria in tap water determined by the ProteoQuant assay. Lett. Appl. Microbiol. 50(1):1-6. doi:10.1111/j.1472-765X.2009.02743.x. PMID:19874479. 
Salter, S.J., Cox, M.J., Turek, E.M., Calus, S.T., Cookson, W.O., Moffatt, M.F., Turner, P., Parkhill, J., Loman, N.J., and Walker, A.W. 2014. Reagent and laboratory contamination can critically impact sequence-based microbiome analyses. BMC Biol. 12;12:87. doi:10.1186/s12915-014-0087-z. PMID:25387460.

Schloss, P.D., Westcott, S.L., Ryabin, T., Hall, J.R., Hartmann, M., Hollister, E.B., Lesniewski, R.A., Oakley, B.B., Parks, D.H., Robinson, C.J., Sahl, J.W., Stres, B., Thallinger, G.G., Van Horn, D.J., and Weber, C.F. 2009. Introducing mothur: Open-source, platform-independent, community-supported software for describing and comparing microbial communities. Appl. Environ. Microbiol. 75(23):7537-7541. doi:10.1128/AEM.01541-09.

PMID:19801464.

Sibley, C.D., Grinwis, M.E., Field, T.R., Eshaghurshan, C.S., Faria, M.M., Dowd, S.E., Parkins, M.D., Rabin, H.R., and Surette, M.G. 2011. Culture enriched molecular profiling of the cystic fibrosis airway microbiome. PLoS One. 6(7):25-27. doi: 10.1371/journal.pone.0022702. PMID:21829484.

Simões, L.C., Simões, M., and Vieira, M.J. 2010. Influence of the diversity of bacterial isolates from drinking water on resistance of biofilms to disinfection. Appl. Environ. Microbiol. 76(19):6673-6679. doi:10.1128/AEM.00872-10. PMID:20693444.

Suwandi, A., Bargen, I., Roy, B., Pils, M.C., Krey, M., Zur Lage, S., Basler, T., Rohde, M., Falk, C.S., Hornef, M.W., Goethe, R., and Weiss, S. 2014. Experimental colitis is exacerbated by concomitant infection with Mycobacterium avium ssp. paratuberculosis. Inflamm. Bowel. Dis. 20(11):1962-1971. doi:10.1097/MIB.0000000000000157. PMID:25144571.

Thomas, J.M., and Ashbolt, N.J. 2011. Do free-living amoebae in treated drinking water systems present an emerging health risk? Environ. Sci. Technol. 45(3):860-869. doi:10.1021/es102876y. PMID:21194220. 
Vagianos, K., Clara, I., Carr, R., Graff, L.A., Walker, J.R., Targownik, L.E., Lix, L.M., Rogala, L., Miller, N., and Bernstein, C.N. 2016. What are adults with inflammatory bowel disease (IBD) eating? A closer look at the dietary habits of a population-cased Canadian IBD cohort. J. Parenter. Enter. Nutr. 40(3):405-11. doi:10.1177/0148607114549254. PMID:25189173.

Wang, Q., Garrity, G.M., Tiedje, J.M., and Cole, J.R. 2007. Naïve Bayesian classifier for rapid assignment of rRNA sequences into the new bacterial taxonomy. Appl. Environ. Microbiol. 73(16):5261-5267. PMID:17586664.

Wang, H., Masters, S., Edwards, M.A., Falkinham, J.O., and Pruden, A. 2014. Effect of disinfectant, water age, and pipe materials on bacterial and eukaryotic community structure in drinking water biofilm. Environ. Sci. Technol. 48(3):1426-1435. doi:10.1021/es402636u. PMID:24401122.

Wei, B., Huang, T., Dalwadi, H., Sutton, C.L., Bruckner, D., and Braun, J. 2002. Pseudomonas fluorescens encodes the Crohn's disease-associated 12 sequence and T-cell superantigen. Infect. Immun. 70(12):6567-6575. PMID:12438326.

Wingender, J., and Flemming, H.C. 2011. Biofilms in drinking water and their role as reservoir for pathogens. Int. J. Hyg. Environ. Health. 214(6):417-423. doi:10.1016/j.ijheh.2011.05.009. PMID:21697011. 
Figure 1. Map of Manitoba, Canada illustrating incidence of IBD. Incidence (number of cases per 100,000 population) of Crohn's disease (top) and ulcerative colitis (bottom) in the province of Manitoba and the City of Winnipeg, Manitoba, Canada, 1990-2001. Results were age-standardized to the 1996 Manitoba population. Adapted with permission from Green et al. (2006).

Figure 2. Plot of alpha-diversity metrics. Plots demonstrate community richness (estimated by ACE and Chao1) and diversity (estimated by Shannon, Simpson and inverse-Simpson) variation observed between A) low and high incidence areas B) sample types C) sample locations and D) water distribution networks. Differences considered significant at $*=P<0.05$.

Figure 3. Phylum-level abundances of the water microbiota. Comparison of phyla abundance as documented between A) low and high incidence areas B) sample types C) sample locations and D) water distribution networks. Phyla are expressed as average abundance. Differences considered significant at $*=P<0.05$.

Figure 4. Genus-level abundances of the water microbiota. Comparison of genera abundance as documented between A) low and high incidence areas B) sample types C) sample locations and D) water distribution networks. Genera are expressed as average abundance. Differences considered significant at $*=P<0.05$

Figure 5. Principal coordinate analysis based on the overall structure of the water microbiota of all samples. Principal coordinate analysis was calculated using Bray-Curtis distances. Each data point represents an individual sample. Colour of data point is indicative of A) incidence area and B) sample type. 

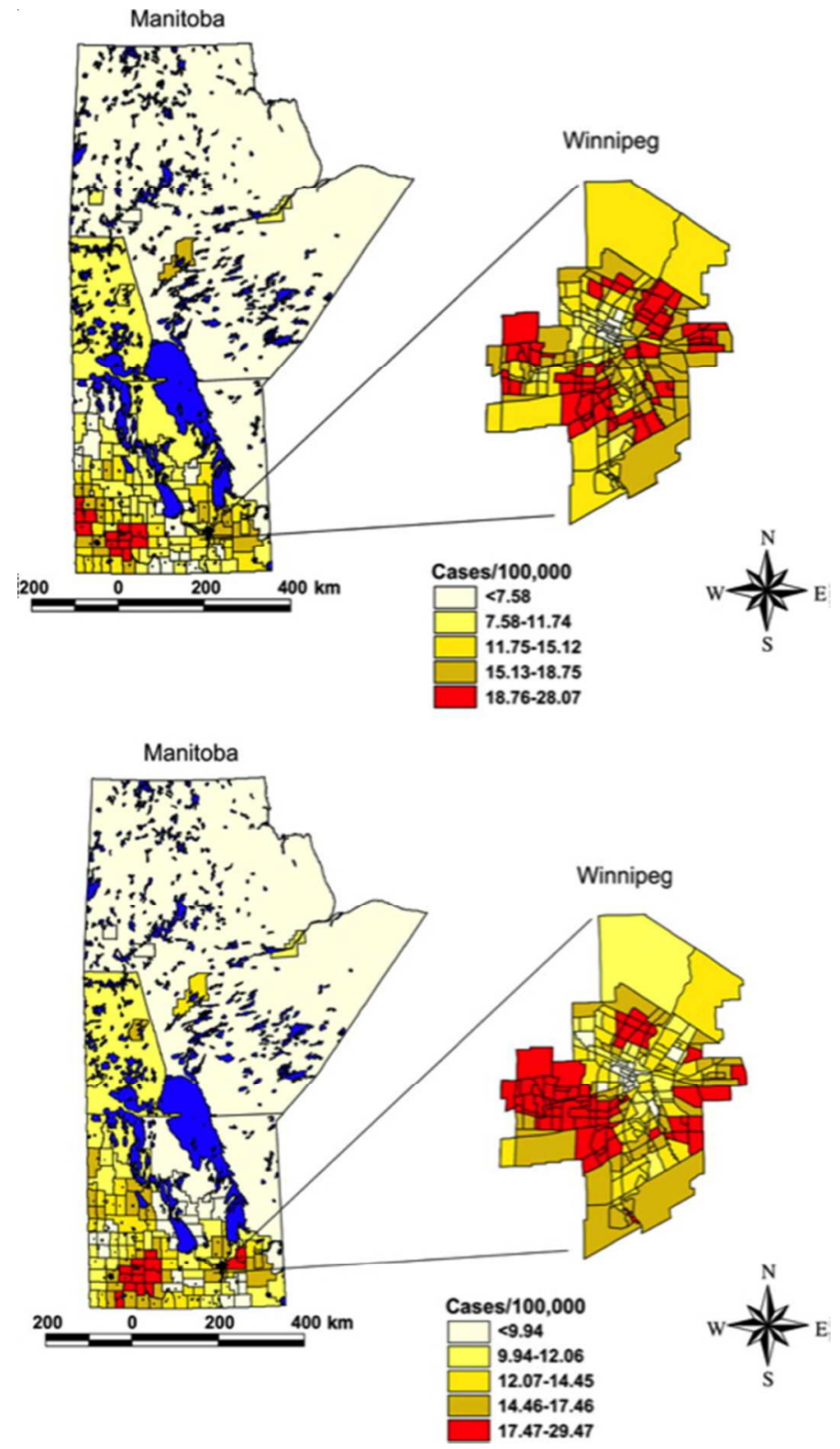

Figure 1. Map of Manitoba, Canada illustrating incidence of IBD. Incidence (number of cases per 100,000 population) of Crohn's disease (top) and ulcerative colitis (bottom) in the province of Manitoba and the City of Winnipeg, Manitoba, Canada, 1990-2001. Results were age-standardized to the 1996 Manitoba population. Adapted with permission from Green et al. (2006). $85 \times 149 \mathrm{~mm}(150 \times 150 \mathrm{DPI})$ 

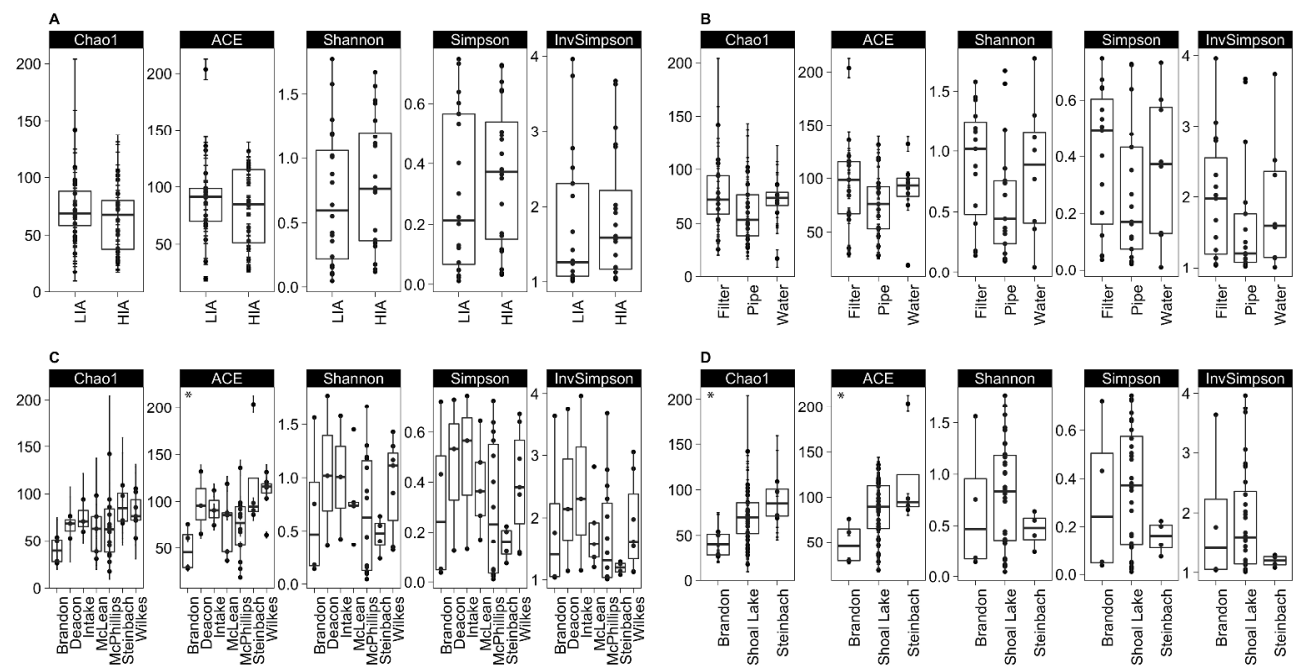

Figure 2. Plot of alpha-diversity metrics. Plots demonstrate community richness (estimated by ACE and Chao1) and diversity (estimated by Shannon, Simpson and inverse-Simpson) variation observed between A) low and high incidence areas $B$ ) sample types $C$ ) sample locations and D) water distribution networks. Differences considered significant at $*=\mathrm{P}<0.05$. $1763 \times 917 \mathrm{~mm}(72 \times 72 \mathrm{DPI})$ 

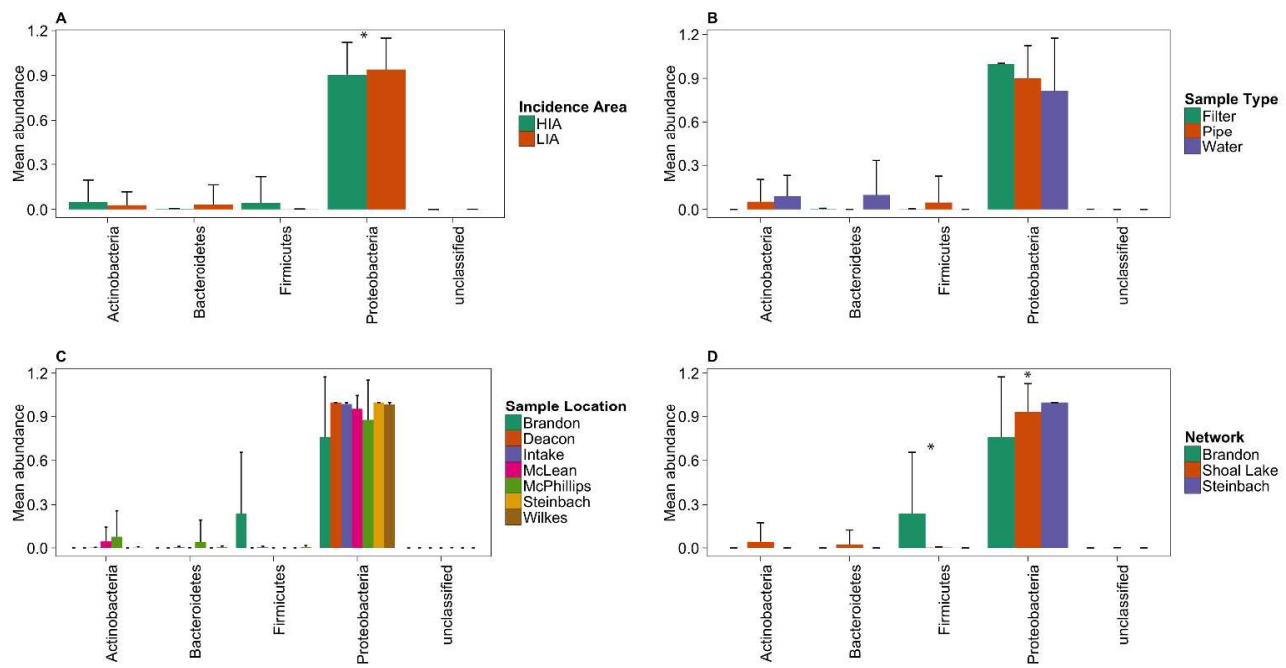

Figure 3. Phylum-level abundances of the water microbiota. Comparison of phyla abundance as documented between A) low and high incidence areas B) sample types C) sample locations and D) water distribution networks. Phyla are expressed as average abundance. Differences considered significant at $*=P<0.05$. $1763 \times 917 \mathrm{~mm}(72 \times 72 \mathrm{DPI})$ 

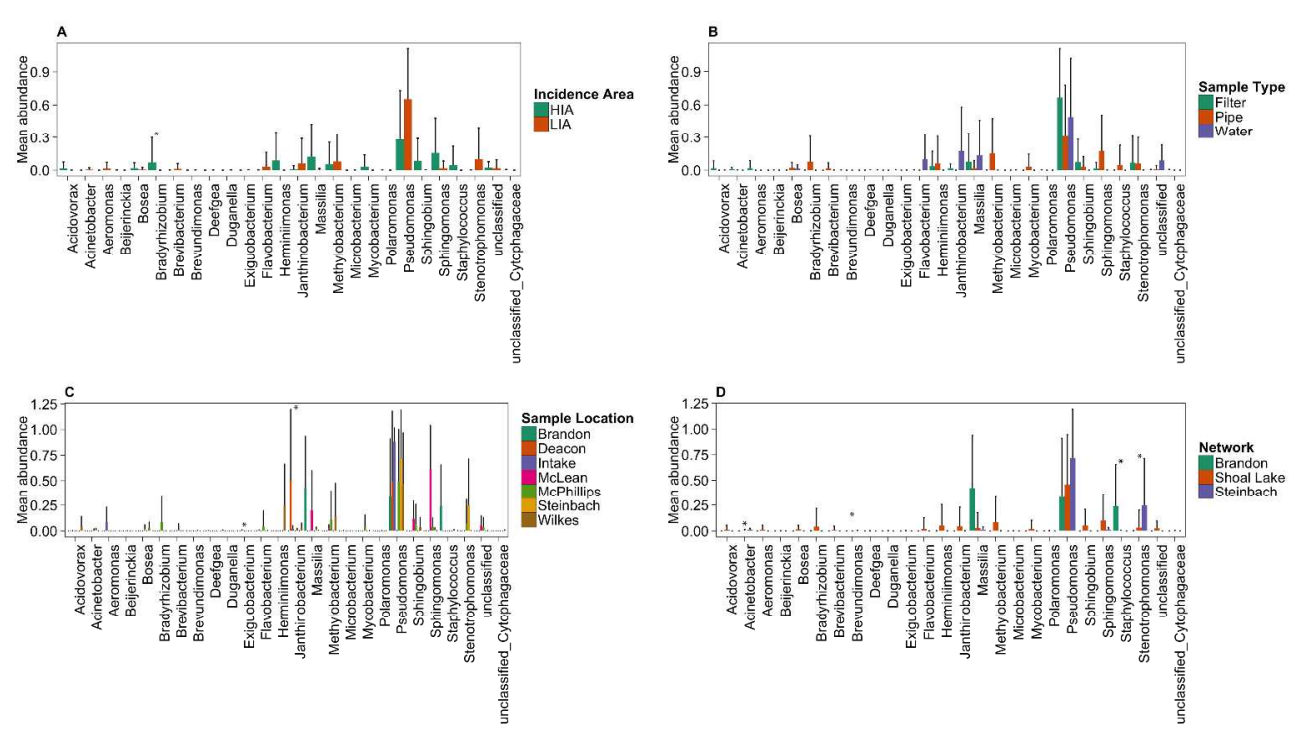

Figure 4. Genus-level abundances of the water microbiota. Comparison of genera abundance as documented between A) low and high incidence areas $B$ ) sample types $C$ ) sample locations and D) water distribution networks. Genera are expressed as average abundance. Differences considered significant at $*=P<0.05$ $1905 \times 1058 \mathrm{~mm}(72 \times 72$ DPI) 

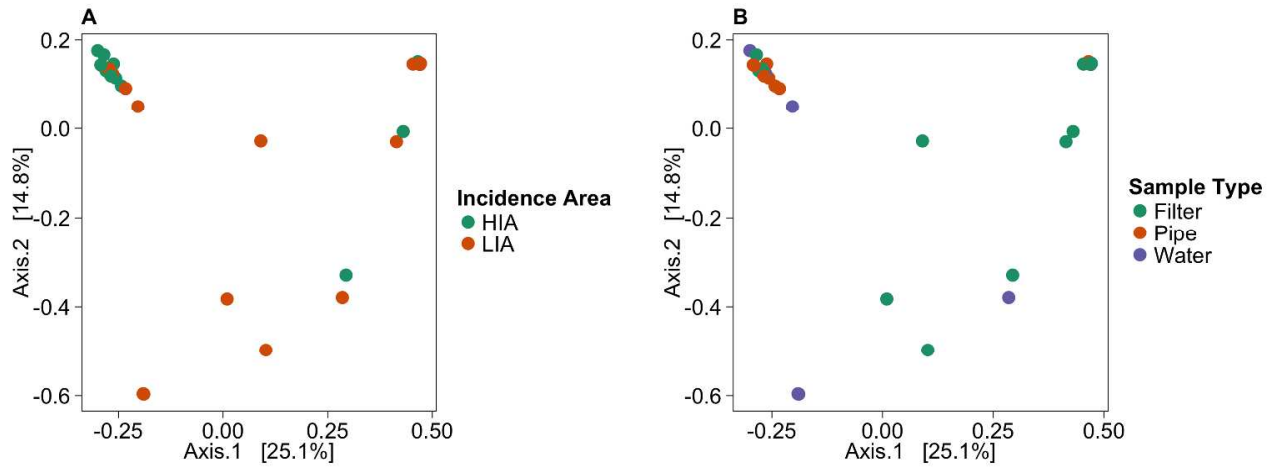

Figure 5. Principal coordinate analysis based on the overall structure of the water microbiota of all samples. Principal coordinate analysis was calculated using Bray-Curtis distances. Each data point represents an individual sample. Colour of data point is indicative of $A$ ) incidence area and B) sample type. $1234 \times 458 \mathrm{~mm}(72 \times 72 \mathrm{DPI})$ 